\title{
DE LA NEUROFOBIA A LA FASCINACIÓN POR LA NEUROLOGÍA
}

\author{
Eduardo Palacios Sánchez MD*
}

Desde su nacimiento más o menos formal en la segunda mitad del siglo XIX, la neurología ha gozado de reconocimiento dentro de la medicina; ha sido catalogada como ciencia de alta complejidad y despertado admiración no sólo de médicos sino de otros profesionales cuyas ciencias, disciplinas y profesiones se han nutrido con los conocimientos por ella proporcionados. El sistema nervioso central, su objeto de estudio, ocupa en la actualidad un puesto privilegiado en la explicación de la conducta, permitiéndonos conocer y comprender en gran parte lo que han sido, lo que son y lo que serán cientos de especies, entre ellas la humana. Así, esta disciplina ofrece un acercamiento excepcional al entendimiento biológico de la conducta normal y patológica.

No obstante, ha sido objeto de prejuicios pues siempre se ha considerado como una de las especialidades más temidas por estudiantes de pre y posgrado, quienes la encuentran difícil e intimidatoria. Quizás esté ligado a ello el estereotipo de los neurólogos descrito por algunos autores como Smith (1999), catalogándolos como hombres brillantes, con extrema facilidad para hablar de la complejidad del cerebro, del diagnóstico y de extraños síndromes.

Recuerdoalgunos estudiantes que comentan que cuando oyen las palabras afasia, apraxia, agnosia piensan que están hablando de algo que no es medicina y prefieren pasar a otro tema. Tal ha sido la ansiedad producida por el aprendizaje de esta ciencia, que hace cerca de dos décadas Jozefowicz acuñó el concepto de neurofobia para referirse "al temor a las ciencias neurológicas y a la neurología clínica que es debido a la inhabilidad de los estudiantes para aplicar sus conocimientos de las ciencias básicas a las situaciones clínicas”. En el estudio de Schon, Hart y Fernández

\footnotetext{
* Jefe del Servicio de Neurología del Hospital de San José. Miembro de la Sociedad de Cirugía de Bogotá. ProfesorTitular de Neurología, Fundación Universitaria de Ciencias de la Salud. Bogotá DC, Colombia.
}

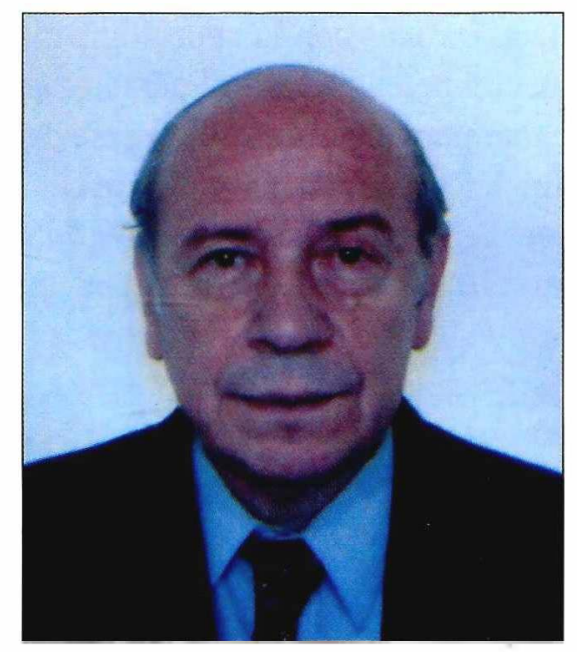

(2002), los médicos identificaron tres aspectos fundamentales que contribuyen a percibirla como una especialidad de intensa dificultad: 1) la necesidad de tener conocimientos sobre las neurociencias básicas y en especial, sobre neuroanatomía; 2) la cantidad, el tipo y la calidad de la enseñanza y 3 ) la dificultad para realizar el examen neurológico.

Durante los últimos años se han realizado esfuerzos para lograr un aprendizaje más amable de la neurología; es el caso de la competencia, el debate y la inclusión del humor como elementos de enseñanza-aprendizaje de la misma (Tan \& tan, 2008); o del programa OFTEN (Open Facilities for Training in European Neurology) European Board of Neurology (Gilhus et al., 2002); o de la telemedicina que utiliza el videolink en tiempo real para entrenar en diagnóstico y plan de manejo de los pacientes neurológicos (Patterson \& Gormley, 2008); o de las sugerencias realizadas por la Asociación de Neurólogos Británicos para la enseñanza de la especialidad en un nuevo currículo integrado (Ford, 2005); o del reconocimiento de la Academia Americana de Neurología sobre la necesidad de educar a los neurólogos en dominios específicos como el de la migraña, las cefaleas (2005) o el de la epilepsia (Ochoa \& Wludyka, 
2008); o del programa basado en la Teoría del Aprendizaje Experiencial (ELC-experiential learning cycle theory) (Emsley, 2009). Sin lugar a dudas, uno de los esfuerzos que cabe resaltar es el del Departamento de Fisiología de la Universidad de Adelaide en Australia, representado por un programa de enseñanza basada en casos (Case Based Teaching-CBT) para integrar las neurociencias básicas con la neurología clínica (Hudson, 2006), competencia central para la especialidad y es posible también que sea esta la fuente del estrés que nutre el prejuicio que se ha consolidado con tanto rigor. Las actitudes de los estudiantes que fueron incluidos en el programa $\mathrm{CBT}$ fue positiva y en especial resultaron facilitadoras de la integración con actividades dirigidas por tutores neurólogos como la práctica, la solución de problemas y el aprendizaje en grupos pequeños. Quienes presentaban neurofobia requirieron de una mayor cantidad de sesiones en el programa.

Lo cierto es que la neurología ha permeado a una importante variedad de especialidades médicas y también a otras ciencias y disciplinas, por lo que el aprendizaje de la teoría sobre el funcionamiento del sistema nervioso central no resulta opcional sino fundamental en muchos escenarios. Sin embargo, tal y como lo plantea la Federación Europea de Sociedades de Neurología (EFNS), tanto ese conocimiento teórico como las habilidades prácticas son componentes básicos del ejercicio actual y óptimo de la neurología (Bartos et al., 2001) y deberán continuarse todos los esfuerzos para que el estudiante y el neurólogo en formación afronten de una manera más adaptativa el reto de aprender esta ciencia que como lo plantean Handel \& Ramagopalan (2009) es, “[...] por su propia naturaleza, la especialidad más fascinante" (p.1764). Entre tanto, el objetivo continuará siendo el de desmitificarla.

\section{Lecturas recomendadas}

Bartos A, Kalvach P, Trost M, Ertsey C, Rejdak K, Popov L, et al. . Postgraduate education in neurology in Central and Eastem Europe. Eur J Neurol. 2001 Nov;8(6):551-8

Ford $\mathrm{H}$. Teaching medical students clinical neurology: a 'young thing's' view Clin. Teach. 2005; 2(2): 115-17.

Emsley H. (2009). Improving undergraduate clinical neurology bedside teaching: opening the magic circle. Clin. Teach. 2009; 6: 172-76.

Gilhus NE, Federico A, Grisold W, Müller L, Lopes lima JM. Open Facilities for Training in European Neurology (OFTEN): a European Board of Neurology initiative. Eur J Neurol. 2002 Jul;9(4):349-52.

Handel AE, Ramagopalan SV. Has Neirology been Demystified?. Lancet. 2009; 373 (9677): 1763-64.

Hudson JN. Linking neuroscience theory to practice to help overcome student fear of neurology. Med Teach. 2006; 28(7): 651-53.

Kommineni M, Finkel AG. Teaching headache in America: survey of neurology chairs and residency directors. Headache. 2005; 45: 862-65.

Ochoa JG, Wludyka P. Randomized Comparison Between Traditional and Traditional Plus Interactive Web-Based Methods for Teaching Seizure Disorders. Teach Leam Med. 2008; 20(2): 114-17.

Patterson V, Gormley S. Teaching neurology by videolink. Med Educ. 2008, 42: $1111-46$

Schon F, Hart P, Fernandez C. (2002). Is clinical neurology really so difficult? J Neurol Neurosurg Psychiatry. 2002; 72:557-59.

Smith R. Editor's choice: neurology for the masses. BMJ. 1999; 319.

Tan NCK, Tan K. Who wants to be a neurologist? An undergraduate quiz. Med Educ. 2008; 42:513-543. 